\title{
Pre-implant left ventricular dimension is not associated with worse outcomes after left ventricular assist device implantation
}

\author{
Amudan J. Srinivasan ${ }^{1 \#}$, Mahbub Jamil ${ }^{2 \#}$, Laura Seese ${ }^{2}$, Ibrahim Sultan ${ }^{2,3}$, Gavin Hickey ${ }^{3}$, Mary E. Keebler ${ }^{3}$, \\ Michael A. Mathier ${ }^{3}$, Arman Kilic ${ }^{2,3}$ \\ ${ }^{1}$ Department of Surgery, University of Pittsburgh Medical Center, Pittsburgh, PA, USA; ${ }^{2}$ Division of Cardiac Surgery, University of Pittsburgh \\ Medical Center, Pittsburgh, PA, USA; ${ }^{3}$ Heart and Vascular Institute, University of Pittsburgh Medical Center, Pittsburgh, PA, USA \\ Contributions: (I) Conception and design: AJ Srinivasan, M Jamil, L Seese, A Kilic; (II) Administrative support: I Sultan, G Hickey, ME Keebler, \\ MA Mathier, A Kilic; (III) Provision of study materials or patients: M Jamil, I Sultan, G Hickey, ME Keebler, MA Mathier, A Kilic; (IV) Collection \\ and assembly of data: AJ Srinivasan, M Jamil, L Seese, A Kilic; (V) Data analysis and interpretation: AJ Srinivasan, M Jamil, L Seese, A Kilic; (VI) \\ Manuscript writing: All authors; (VII) Final approval of manuscript: All authors. \\ \#These authors contributed equally to this work as co-first authors. \\ Correspondence to: Arman Kilic, MD. Division of Cardiac Surgery, University of Pittsburgh Medical Center, 200 Lothrop Street, Suite C-700, \\ Pittsburgh, PA 15213, USA. Email: kilica2@upmc.edu.
}

Background: Left ventricular dimension has the potential to impact clinical outcomes following implantation of left ventricular assist devices (LVAD). We investigated the effect of pre-implant left ventricular end-diastolic diameter (LVEDD) on outcomes following LVAD implantation.

Methods: Patients implanted with a continuous-flow LVAD between 2004 and 2018 at a single institution were included. The primary outcome was death while on LVAD support. Secondary outcomes included adverse event rates such as renal failure requiring dialysis, device thrombosis, and right ventricular failure. The LVEDD measurements were dichotomized using restricted cubic splines and threshold regression. Survival was determined using Kaplan-Meier estimates. Multivariable logistic regression was used to determine risk-adjusted mortality based on LVEDD.

Results: A total of 344 patients underwent implantation of a continuous flow LVAD during the study period. The optimal cut point for LVEDD was $65 \mathrm{~mm}$, with $126(36.6 \%)$ subjects in the $<65 \mathrm{~mm}$ group and $165(48.0 \%)$ in the $>65 \mathrm{~mm}$ group. The LVEDD $<65 \mathrm{~mm}$ group was older, had more females, higher incidence of diabetes, more pre-implant mechanical ventilation, and more admissions for acute myocardial infarctions (all, $\mathrm{P}<0.05$ ). Importantly, post-implant adverse events were similar between the groups (all, $\mathrm{P}>0.05$ ). Risk-adjusted survival at 1-year ( $\mathrm{OR} 1.3,95 \% \mathrm{CI}$ : 0.6-2.5, P=0.53) was also comparable between the groups. Furthermore, incremental increases in LVEDD when modeled as a continuous variable did not impact overall mortality (OR 0.98, 95\% CI: 0.9-1.0, P=0.09).

Conclusions: Preoperative LVEDD was not associated with rates of major morbidities or mortality following LVAD implantation.

Keywords: Left ventricular end-diastolic diameter (LVEDD); ventricular assist device; mechanical circulatory support; advanced heart failure

Submitted Aug 30, 2020. Accepted for publication Dec 10, 2020.

doi: $10.21037 /$ jtd-20-2778

View this article at: https://dx.doi.org/10.21037/jtd-20-2778

\section{Introduction}

Left ventricular assist devices (LVADs) are a mainstay therapy in the management of patients with advanced heart failure, both as a bridge to heart transplant in eligible patients and as destination therapy $(1,2)$. Although utilization of LVADs has increased in recent years, adverse 
events that are both directly and indirectly related to the device continue to be a limiting factor in improving quality of life or survival for some patients (3). Numerous studies have identified predictors of poor outcomes following LVAD implantation, such as advanced age or poor baseline right ventricular function (4). Comparatively less work has been done thus far to determine the influence of intrinsic mechanical and structural properties of the heart on LVAD function and overall patient outcomes. It has been suggested by both computational fluid dynamic modeling (5) and registry studies $(6,7)$ that smaller left ventricular dimension, as measured by left ventricular end-diastolic dimension (LVEDD), is associated with both right ventricular failure (8) while on LVAD therapy and worse overall survival. We sought to evaluate whether preoperative LVEDD would be associated with major morbidity and mortality in a contemporary cohort of patients supported with continuous flow LVADs.

We present the following article in accordance with the STROBE reporting checklist (available at https://dx.doi. org/10.21037/jtd-20-2778).

\section{Methods}

\section{Data source and study population}

Data was sourced from a prospectively-maintained institutional database that encompasses outcomes for all patients undergoing implantation of LVADs at a multihospital institution. Patients undergoing continuousflow LVAD implantation for either a bridge-to-transplant or destination therapy strategy at a tertiary academic referral center between 2004-2018 were included in this analysis. All data points were abstracted from the electronic health record (Cerner Corporation, Kansas City, MO, USA), and LVEDD data was obtained from preoperative echocardiographic reports prior to implantation. The study was conducted in accordance with the Declaration of Helsinki (as revised in 2013). The study was approved by institutional review board of the University of Pittsburgh (No: Study18120143) under an approved waiver of informed consent for this retrospective analysis.

\section{Outcomes}

The primary outcome for this study was 30-day and 1-year all-cause mortality. Secondary outcomes included major systemic, surgical, and device-related complications.
Systemic complications included infection, stroke, right ventricular failure, renal failure requiring dialysis, or hepatic failure. Device complications included device thrombosis, hemolysis, device dysfunction, and device explant. Surgical complications included bleeding, reoperation for all causes and reoperation for tamponade. Additionally, another secondary outcome was successful bridging to cardiac transplantation.

\section{Data analysis}

All continuous data are presented as mean \pm standard deviation or median [interquartile range (IQR)] and all categorical data as number (percentage). Normally distributed data were compared with two-sided Student's $t$-tests while non-Gaussian distributions were evaluated using quantile regression. Threshold regression analysis, adjusted for gender and body surface area, and restricted cubic splines analysis was used to determine the optimal cut point for LVEDD. Kaplan-Meier estimates were used to compare longitudinal survival. Multivariable logistic regression (univariate inclusion criteria two-tailed $\mathrm{P}<0.05$ ) was used to determine risk-adjusted mortality at 1 year based on LVEDD. Significance was defined as two-tailed $\mathrm{P}<0.05$.

We evaluated the missing data and determined it to be missing at random. In order to do this, we initially evaluated the pattern of missingness and then the mechanism of missingness. To do this, we started with Little's hypothesis test to determine if the data was missing completely at random and found that it was not. Then we evaluated if missingness was associated with other variables within the data set including LVAD type, bridging strategy, and gender. We then evaluated the variables that had monotone missingness with the Listing and Schlittgen test, which demonstrated similar conclusions to Little's test indicating that the missing data was not MCAR. Finally, we used Fairclough logistic regression where covariates associated with missingness in patients who died from multisystem organ failure were included and the missing data was found to be missing at random.

\section{Results}

\section{Patient characteristics}

A total of 344 patients underwent implantation of a continuous flow LVAD during the study period. The 


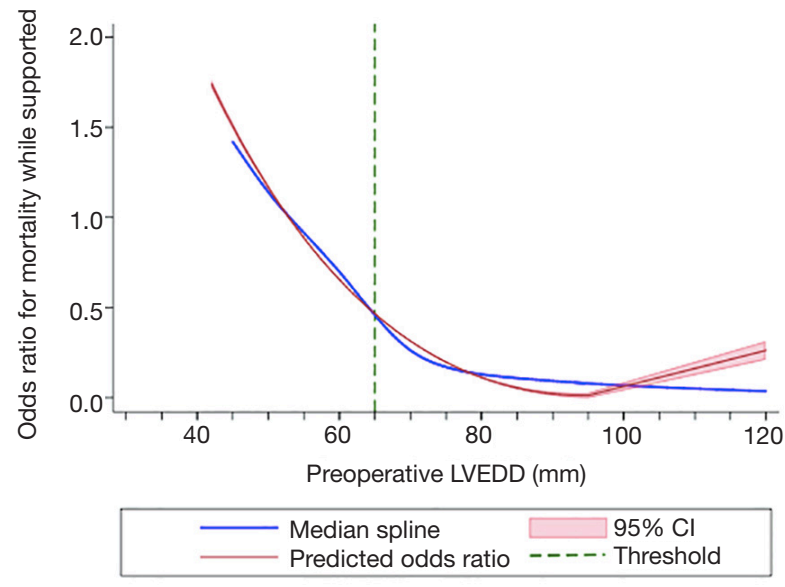

Figure 1 Smoothed median cubic spline plot demonstrating odds ratio for mortality while supported on an LVAD device based on preoperative LVEDD with threshold value indicated at $65 \mathrm{~mm}$. LVEDD, left ventricular diastolic diameter; LVAD, left ventricular assist device.

optimal cut point for LVEDD was $65 \mathrm{~mm}$, and patients were stratified into two cohorts with $126(36.6 \%)$ in the $<65 \mathrm{~mm}$ cohort and $165(63.4 \%)$ in the $>65 \mathrm{~mm}$ cohort. Derivation of the $65 \mathrm{~mm}$ cut point for this analysis is demonstrated in Figure 1. Those in the $<65 \mathrm{~mm}$ cohort were older (59.1 vs. 55.4 years, $\mathrm{P}=0.013)$, more frequently female $(21 \%$ vs. $13 \%, \mathrm{P}=0.048)$, and had a lower body surface area $\left(2.05 \pm 0.29\right.$ vs. $\left.2.12 \pm 0.30 \mathrm{~m}^{2}, \mathrm{P}=0.030\right)$. The $<65 \mathrm{~mm}$ cohort additionally had a greater burden of comorbid conditions as evidenced by the higher incidence of diabetes $(50 \%$ vs. $34 \%, \mathrm{P}=0.009)$, higher baseline serum creatinine $(1.60 \pm 0.65$ vs. $1.43 \pm 0.58 \mathrm{mg} / \mathrm{dL}, \mathrm{P}=0.023)$, and more frequent pre-implant mechanical ventilation $(5.3 \%$ vs. $0.8 \%, \mathrm{P}=0.035$ ). Patients in the $<65 \mathrm{~mm}$ group were more likely to have been admitted for acute myocardial infarction $(14 \%$ vs. $6 \%, \mathrm{P}=0.04)$ but were less likely to have an implanted cardioverter-defibrillator $(69.8 \%$ vs. $83.6 \%$, $\mathrm{P}=0.005)$. There were not significant differences in the underlying cardiac diagnoses/indications for LVAD therapy between the two groups, with ischemic or idiopathic dilated cardiomyopathy comprising the majority in both cohorts. Both groups were equally likely to have required inotropic support in the preoperative period, though the $<65 \mathrm{~mm}$ group was more likely to have required a dopamine infusion ( $8.5 \%$ vs. $2.3 \%, \mathrm{P}=0.031)$. The use of preimplant intraaortic balloon pumps was similar between the two groups, and there was no significant between-group difference in hemodynamic parameters such as central venous pressure, mean pulmonary artery pressure, pulmonary capillary wedge pressure, and transpulmonary gradient (all, $\mathrm{P}>0.05$ ). Furthermore, the different LVAD device types included in the study were found to be evenly distributed between the groups. A complete listing of baseline characteristics is provided (Table 1), as well as a distribution of LVEDD values (Table 2).

\section{Post-implant adverse events}

There was no significant difference between the $<65$ and $>65 \mathrm{~mm}$ groups in post-implant mortality at both 30 days ( $4.2 \%$ vs. $2.8 \%, \mathrm{P}=0.48)$ and 1 year $(45 \%$ vs. $33 \%, \mathrm{P}=0.98)$. Compared to the $>65 \mathrm{~mm}$ group, the $<65 \mathrm{~mm}$ group had similar incidence of infection $(46.4 \%$ vs. $44.5 \%, \mathrm{P}=0.79)$, dialysis-dependent renal failure $(20.2 \%$ vs. $13.4 \%, \mathrm{P}=0.20)$, hepatic dysfunction $(2.4 \%$ vs. $3.4 \%, \mathrm{P}=0.68)$, stroke $(17.9 \%$ vs. $12.6 \%, \mathrm{P}=0.30)$, and right ventricular dysfunction ( $13.1 \%$ vs. $10.9 \%, \mathrm{P}=0.64)$. There were additionally similar rates of device complications between the two groups, including device dysfunction $(13.1 \%$ vs. $16.0 \%, \mathrm{P}=0.57)$, device thrombosis $(3.6 \%$ vs. $3.4 \%, \mathrm{P}=0.94)$, hemolysis (8.3\% vs. $5.0 \%, \mathrm{P}=0.35)$, and device explantation $(7.1 \%$ vs. $10.9 \%, \mathrm{P}=0.35)$. Finally, the rates of surgical complications were similar between the two cohorts, with no significant difference in bleeding ( $36.9 \%$ vs. $36.1 \%, \mathrm{P}=0.91)$, reoperation for tamponade $(19.0 \%$ vs. $10.9 \%, \mathrm{P}=0.10)$, and all-cause reoperation $(25.0 \%$ vs. $28.6 \%, \mathrm{P}=0.57)$. In the $<65 \mathrm{~mm}$ group, $30.1 \%$ of subjects proceeded to cardiac transplantation compared to $40.5 \%$ in the $>65 \mathrm{~mm}$ group, though this difference was not statistically significant $(\mathrm{P}=0.11)$. The complete post-implant data can be found in Table 3, and Kaplan-Meier survival curves stratified by LVEDD are depicted in Figure 2.

\section{Multivariable logistic regression}

A low LVEDD was not a significant predictor of 1-year (OR 1.37, 95\% CI: 0.71-2.64, P=0.34) unadjusted mortality when compared to the reference high-LVEDD group. Furthermore, when risk-adjusted, low LVEDD also did not predict 1-year (OR 1.26, 95\% CI: 0.61-2.54, $\mathrm{P}=0.53)$ mortality. Instead, traditional risk-factors such as preoperative mechanical ventilation (OR 1.42, 95\% CI: 1.06-1.88, $\mathrm{P}=0.02$ ), dialysis dependence (OR 2.05, 95\% CI: 1.78-2.18, $\mathrm{P}=0.01)$, and increasing serum bilirubin (OR $1.29,95 \% \mathrm{CI}: 1.01-1.91, \mathrm{P}=0.03$ ), were found to be drivers 
Table 1 Baseline characteristics stratified by preoperative LVEDD

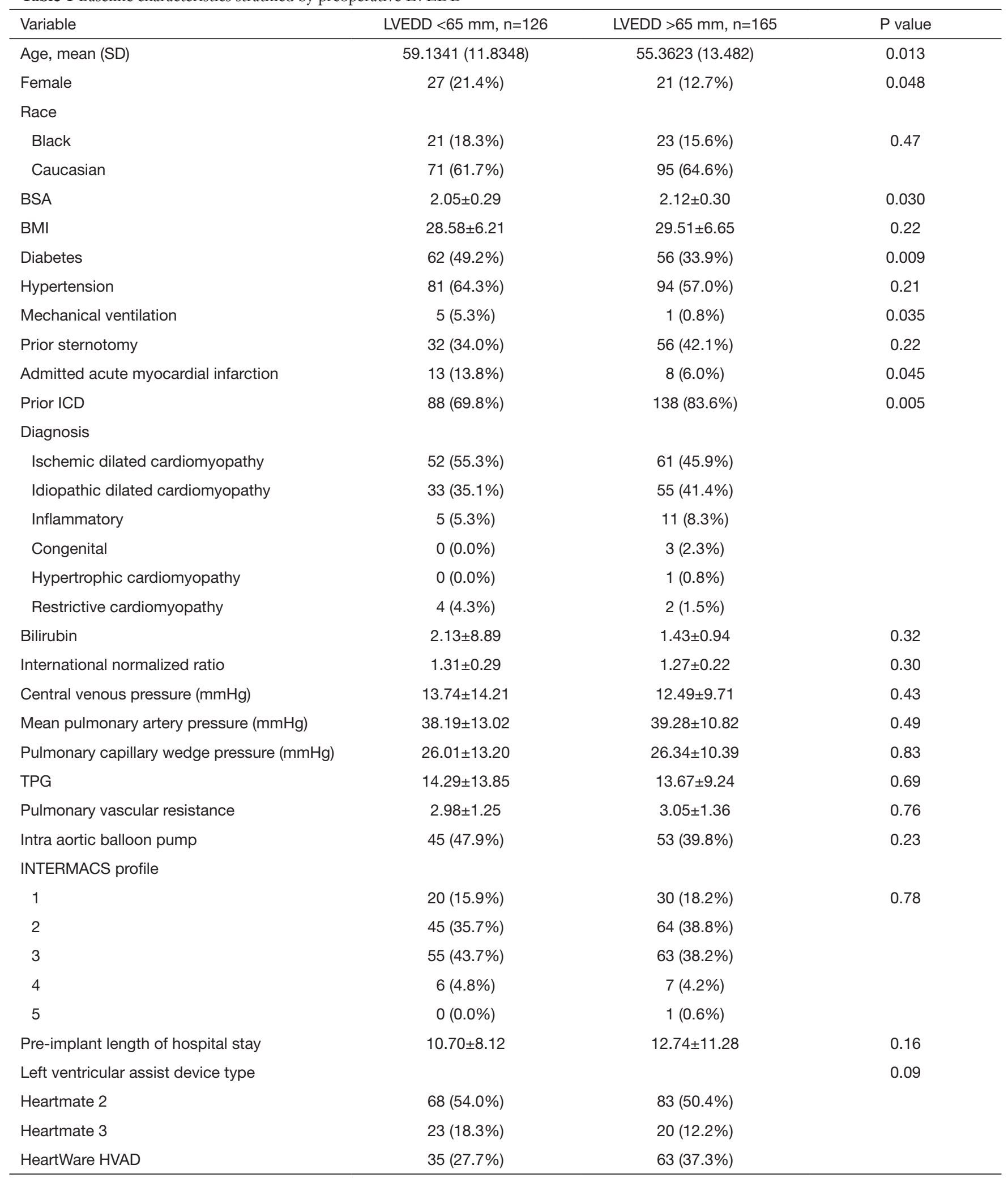

LVEDD, left ventricular diastolic diameter. 
of mortality. LVAD type was not found to impact mortality or to interact with the low- or high-LVEDD groups. When LVEDD was treated as a continuous, increasing variable, there remained no significant impact on risk-adjusted mortality (OR 0.98, 95\% CI: 0.94-1.00, P=0.09). Complete regression results can be found in Table 4 .

\section{Discussion}

Patient selection for LVAD therapy can be challenging, with multiple factors including overall surgical candidacy

Table 2 Distribution of preoperative LVEDD values

\begin{tabular}{lc}
\hline Preoperative LVEDD & $\mathrm{n}(\%)$ \\
\hline $42-49 \mathrm{~mm}$ & $6(2.05 \%)$ \\
$50-55 \mathrm{~mm}$ & $18(6.14 \%)$ \\
$56-70 \mathrm{~mm}$ & $148(50.5 \%)$ \\
$70-90 \mathrm{~mm}$ & $115(39.25 \%)$ \\
$91-121 \mathrm{~mm}$ & $6(2.05 \%)$ \\
\hline
\end{tabular}

LVEDD, left ventricular diastolic diameter. and timing of therapy often being debated by members of multidisciplinary teams. Such teams rely on assessing potential risk factors for adverse outcomes when making decisions regarding LVAD candidacy. One set of such factors are the physical ventricular dimensions surrounding device implantation. Theoretically, a smaller left ventricular chamber as evidenced by a smaller LVEDD would lead to more difficulty in appropriately unloading the ventricle and in sustaining unobstructed laminar flow into the inflow cannula of the device. Nevertheless, the existing literature is equivocal regarding the impact of $\mathrm{LV}$ size on LVAD outcomes, and this led us to evaluate our institutional experience regarding LVAD implantation to identify what effect, if any, preoperative LVEDD had on postimplantation outcomes.

\section{Study findings}

This analysis demonstrates that preoperative LVEDD is not associated with worse outcomes following LVAD implantation in a single-institution cohort of advanced heart failure patients. Notably, this finding persists despite

Table 3 Post-implant adverse events

\begin{tabular}{lccc}
\hline Event & LVEDD $<65 \mathrm{~mm}, \mathrm{n}=126$ & LVEDD $>65 \mathrm{~mm}, \mathrm{n}=165$ & P value \\
\hline Infection & $39(46.4 \%)$ & $53(44.5 \%)$ & 0.79 \\
Bleeding & $31(36.9 \%)$ & $43(36.1 \%)$ & 0.91 \\
Reoperation (all-cause) & $21(25.0 \%)$ & $34(28.6 \%)$ & 0.57 \\
Reoperation for tamponade & $16(19.0 \%)$ & $13(10.9 \%)$ & 0.10 \\
Renal failure & $17(20.2 \%)$ & $16(13.4 \%)$ & 0.20 \\
Hepatic dysfunction & $2(2.4 \%)$ & $4(3.4 \%)$ & 0.68 \\
Stroke & $15(17.9 \%)$ & $15(12.6 \%)$ & 0.30 \\
Right ventricular failure & $11(13.1 \%)$ & $13(10.9 \%)$ & 0.64 \\
RVAD & $2(2.4 \%)$ & $6(5.0 \%)$ & 0.34 \\
Device dysfunction & $11(13.1 \%)$ & $19(16.0 \%)$ & 0.57 \\
Device thrombosis & $3(3.6 \%)$ & $4(3.4 \%)$ & 0.94 \\
Hemolysis & $7(8.3 \%)$ & $6(5.0 \%)$ & 0.35 \\
$30-$ day mortality & $7(4.2 \%)$ & $3(2.8 \%)$ & 0.48 \\
1-year mortality & $23(18.3 \%)$ & $26(15.8 \%)$ & 0.57 \\
Overall mortality & $51(40.5 \%)$ & $56(33.9 \%)$ & 0.25 \\
Device explanted & $6(7.1 \%)$ & $13(10.9 \%)$ & 0.35 \\
\hline
\end{tabular}

LVEDD, left ventricular diastolic diameter. 

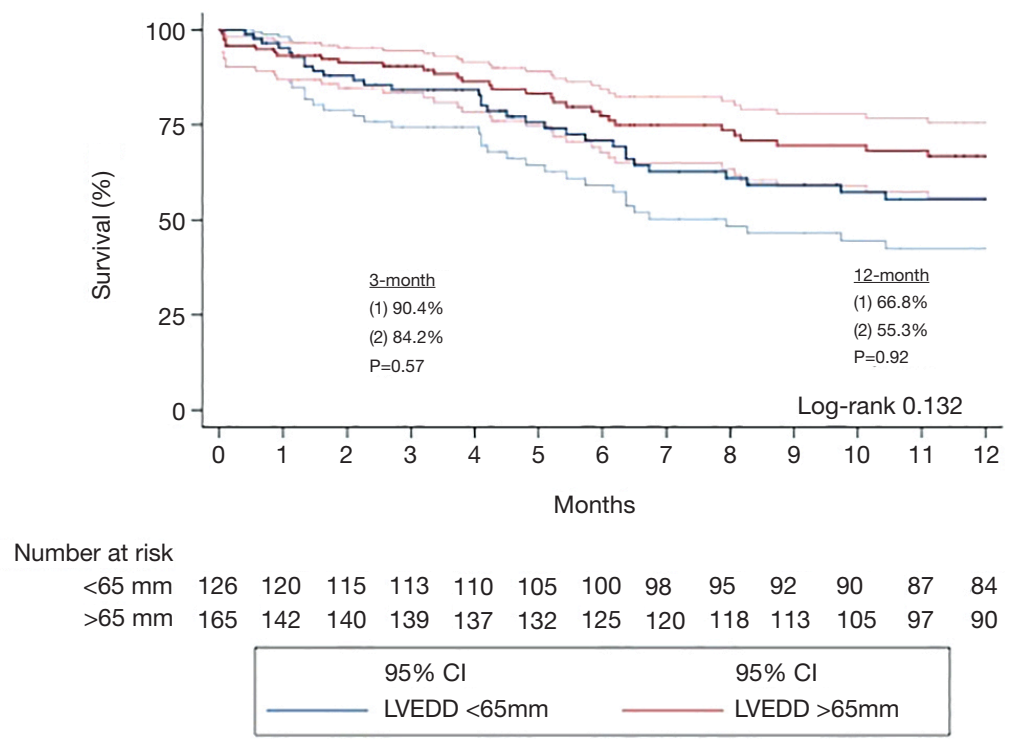

Figure 2 Kaplan-Meier estimates for survival while on device stratified by left ventricular end diastolic diameter. LVEDD, left ventricular diastolic diameter.

Table 4 Univariate and multivariable logistic regression demonstrating the impact of LVEDD on mortality during LVAD support

\begin{tabular}{|c|c|c|c|}
\hline Variable & Odds ratio & $95 \%$ confidence interval & $P$ values \\
\hline \multicolumn{4}{|l|}{ Preoperative LVEDD } \\
\hline Greater than $65 \mathrm{~mm}$ & Reference & Reference & Reference \\
\hline Less than $65 \mathrm{~mm}$ & 1.37 & $0.71,2.64$ & 0.34 \\
\hline \multicolumn{4}{|l|}{ Preoperative LVEDD } \\
\hline Greater than $65 \mathrm{~mm}$ & Reference & Reference & Reference \\
\hline Less than $65 \mathrm{~mm}$ & 1.26 & $0.61,2.54$ & 0.53 \\
\hline Bilirubin (increasing, per mg/dL) & 1.29 & $1.01,1.91$ & 0.03 \\
\hline \multicolumn{4}{|l|}{ Unadjusted overall mortality } \\
\hline Preoperative LVEDD (increasing, per mm) & 0.97 & $0.95,0.99$ & 0.03 \\
\hline \multicolumn{4}{|l|}{ Risk-adjusted overall mortality ${ }^{\ddagger}$} \\
\hline Preoperative LVEDD (increasing, per mm) & 0.98 & $0.94,1.00$ & 0.09 \\
\hline
\end{tabular}

${ }^{\dagger}$, variables included in the multivariable model at 1-year for risk-adjustment: gender, race, bilirubin level, implant year, intermacs profile, left ventricular assist device type; ${ }^{\ddagger}$, variables included in the overall mortality model for risk-adjustment: gender, race, bilirubin level, implant year, prealbumin level, intermacs profile, mean pulmonary artery pressure, left ventricular assist device type. LVEDD, left ventricular diastolic diameter; LVAD, left ventricular assist device. 
the $<65 \mathrm{~mm}$ cohort being a sicker group at baseline with a more substantial burden of comorbidities. This cohort was older, had a higher percentage of patients with diabetes and elevated creatinine, and more frequently required preoperative mechanical ventilation, all of which have been associated with worse outcomes in the LVAD population $(3,9)$.

There was additionally no association between preoperative LVEDD and postoperative device complications, which can be a major concern when implanting an LVAD in a smaller left ventricle. Joyce and colleagues had previously described left ventricular size change following LVAD implantation as a potential predictor of device thrombosis in axial-flow devices, hypothesizing that impairment in left ventricular unloading represented a degree of patient-pump mismatch that ultimately manifested as device thrombosis (10). Shah and colleagues noted that a larger preoperative LVEDD was associated with higher risk of pump thrombosis in axialflow versus centrifugal devices, and was associated with a lower odds of risk-adjusted mortality for all types of LVADs (11). Furthermore, concerns for impairment in device inflow leading to pump thrombosis have been validated by studies evaluating anatomic and surgical factors such as inflow cannula angle (12), left ventricular apex position (13), and smaller left ventricular size (5). Contradistinctively, preoperative left ventricular size did not appear to associate significantly with device thrombosis in our study population.

Additionally, in our study population, preoperative LVEDD measurements did not predict postoperative right ventricular failure or need for subsequent right ventricular mechanical support. There has been an extensive body of work evaluating echocardiographic predictors of right ventricular dysfunction following LVAD implantation, and several studies have correlated LVEDD (14) or comparison of right ventricular and left ventricular dimensions $(8,15)$ to postoperative right ventricular dysfunction. Specifically, many clinicians suspect that potentially inadequate unloading of the left ventricle by LVADs placed in small chambers can lead to pulmonary congestion, elevated pulmonary pressures, higher right heart afterload, and subsequent right heart failure. Though our data does not include a thorough analysis of right-sided echocardiographic parameters, there was no association between the preoperative left ventricular dimension and subsequent right ventricular failure or need for right ventricular assist device use.
Surgical nuances in implantation technique become particularly relevant in patients with small left ventricular dimensions. The inflow cannula, whether placed at the true apex or not, should be parallel to the interventricular septum and directed towards the mitral valve. This orientation should be confirmed after chest closure. This is particularly important in patients with a small chest cavity as the inflow cannula can shift position in relation to the septum. In a small left ventricular cavity there is less physical space for the inflow cannula and therefore active suction on the septum with potential ingestion of muscle is a possibility if the orientation is suboptimal. While some surgeons may choose different device types based on ventricular size, our data suggested that there were no differences in outcomes based on device type in the high- or low- LVEDD groups.

\section{Prior studies evaluating left ventricular size in LVAD populations}

Kawabori and colleagues reviewed their institutional experience with patients implanted with continuous-flow LVADs between 2003 and 2016, stratifying this population into two cohorts based on a LVEDD cut point of $5.5 \mathrm{~cm}$. When evaluating long-term outcomes, they ultimately found that smaller LVEDD predicted worse overall survival in patients implanted with an axial-flow device (Thoratec Heartmate II), though this association was not observed in patients who received a centrifugal-flow device (Heartware HVAD) (7). These findings led the authors to suggest use of the HVAD in patients with a smaller left ventricle. In a subsequent analysis of their institution's Heartmate II recipients, they confirmed their original findings, noting that patients receiving a Heartmate II who had a LVEDD under $6.0 \mathrm{~cm}$ had lower overall survival and an elevated risk of postoperative stroke (6).

It is additionally possible that some of the findings of elevated risk in small LVEDD patients on LVAD therapy stems from concerns for worse outcomes and resultant decreased LVAD use in patients with restrictive and hypertrophic cardiomyopathies, who frequently have smaller ventricular dimensions due to proliferative myocardium. Initial small-cohort institutional studies found evidence of generally comparable mortality between restrictive and dilated cardiomyopathy patients undergoing LVAD therapy, though Topilsky noted higher rates of right ventricular failure and prolonged inotrope use in restrictive cardiomyopathy patients (16), and Grupper 
noted significantly increased mortality in the subgroup of restrictive cardiomyopathy patients with LVEDD $<4.6 \mathrm{~cm}$ (17). A subsequent INTERMACS registry study of patients with restrictive and hypertrophic cardiomyopathy on LVAD therapy recapitulated the mortality and major morbidity findings of the Grupper study, and again noted decreased survival in the subset of patients with small left ventricles $(\mathrm{LVEDD}<5.0 \mathrm{~cm})(18)$.

\section{Limitations}

There are several limitations to our study. First, its design as a single-center retrospective analysis is open to potential confounding and bias due to institution-specific unmeasured variables. The study is additionally limited by the fact that subjects were implanted with one of several device types, including Heartmate 3 (Abbott Laboratories, Chicago, IL, USA), Heartmate 2 (Abbott Laboratories, Chicago, IL, USA), and Heartware HVAD (Medtronic, Minneapolis, MN, USA). With the advent of centrifugal flow devices, the Heartmate 2 has seen a downtrend in clinical use, and this represents one limitation of our study. Furthermore, certain devices or classes of device might be more vulnerable to malfunction or may predispose patients to major morbidity in the setting of small LV size, though this is not identified in the aggregate viewpoint of our analysis. Alongside this point, it is not clear if this data will be generalizable to future devices, as unique device designs will undoubtedly have different mechanical properties and dimensions that would require dedicated analyses to determine the impact of $\mathrm{LV}$ size on device function and outcomes.

\section{Conclusions}

In an institutional cohort of patients supported with continuous-flow LVADs, preoperative LVEDD was not predictive of mortality or major morbidity following LVAD implantation.

\section{Acknowledgments}

Funding: None.

\section{Footnote}

Reporting Checklist: The authors have completed the STROBE reporting checklist. Available at https://dx.doi. org/10.21037/jtd-20-2778
Data Sharing Statement: Available at https://dx.doi. org/10.21037/jtd-20-2778

Conflicts of Interest: All authors have completed the ICMJE uniform disclosure form (available at https://dx.doi. org/10.21037/jtd-20-2778). AK reports that he is on the medical advisory board for Medtronic, Inc. The other authors have no conflicts of interest to declare.

Ethical Statement: The authors are accountable for all aspects of the work in ensuring that questions related to the accuracy or integrity of any part of the work are appropriately investigated and resolved. The study was conducted in accordance with the Declaration of Helsinki (as revised in 2013). The study was approved by institutional review board of the University of Pittsburgh (No: Study18120143) under an approved waiver of informed consent for this retrospective analysis.

Open Access Statement: This is an Open Access article distributed in accordance with the Creative Commons Attribution-NonCommercial-NoDerivs 4.0 International License (CC BY-NC-ND 4.0), which permits the noncommercial replication and distribution of the article with the strict proviso that no changes or edits are made and the original work is properly cited (including links to both the formal publication through the relevant DOI and the license). See: https://creativecommons.org/licenses/by-nc-nd/4.0/.

\section{References}

1. Rose EA, Gelijns AC, Moskowitz AJ, et al. Long-term use of a left ventricular assist device for end-stage heart failure. N Engl J Med 2001;345:1435-43.

2. Takeda K, Takayama H, Kalesan B, et al. Long-term outcome of patients on continuous-flow left ventricular assist device support. J Thorac Cardiovasc Surg 2014;148:1606-14.

3. Holman WL, Kormos RL, Naftel DC, et al. Predictors of death and transplant in patients with a mechanical circulatory support device: a multi-institutional study. J Heart Lung Transplant 2009;28:44-50.

4. Gustafsson F, Rogers JG. Left ventricular assist device therapy in advanced heart failure: patient selection and outcomes. Eur J Heart Fail 2017;19:595-602.

5. Chivukula VK, Beckman JA, Prisco AR, et al. Small Left Ventricular Size Is an Independent Risk Factor for Ventricular Assist Device Thrombosis. ASAIO J 
2019;65:152-9.

6. Kawabori M, Kurihara C, Conyer R, et al. A left ventricular end-diastolic dimension less than $6.0 \mathrm{~cm}$ is associated with mortality after implantation of an axialflow pump. J Thorac Cardiovasc Surg 2019;157:2302-10.

7. Kawabori M, Kurihara C, Sugiura T, et al. ContinuousFlow Left Ventricular Assist Device Implantation in Patients With a Small Left Ventricle. Ann Thorac Surg 2018;105:799-806.

8. Vivo RP, Cordero-Reyes AM, Qamar U, et al. Increased right-to-left ventricle diameter ratio is a strong predictor of right ventricular failure after left ventricular assist device. J Heart Lung Transplant 2013;32:792-9.

9. Topkara VK, Coromilas EJ, Garan AR, et al. Preoperative Proteinuria and Reduced Glomerular Filtration Rate Predicts Renal Replacement Therapy in Patients Supported With Continuous-Flow Left Ventricular Assist Devices. Circ Heart Fail 2016;9:e002897.

10. Joyce E, Stewart GC, Hickey M, et al. Left ventricular dimension decrement index early after axial flow assist device implantation: A novel risk marker for late pump thrombosis. J Heart Lung Transplant 2015;34:1561-9.

11. Shah P, Birk S, Maltais S, et al. Left ventricular assist device outcomes based on flow configuration and preoperative left ventricular dimension: An Interagency Registry for Mechanically Assisted Circulatory Support

Cite this article as: Srinivasan AJ, Jamil M, Seese L, Sultan I, Hickey G, Keebler ME, Mathier MA, Kilic A. Pre-implant left ventricular dimension is not associated with worse outcomes after left ventricular assist device implantation. J Thorac Dis 2021;13(9):5458-5466. doi: 10.21037/jtd-20-2778
Analysis. J Heart Lung Transplant 2017;36:640-9.

12. Aliseda A, Chivukula VK, Mcgah P, et al. LVAD Outflow Graft Angle and Thrombosis Risk. ASAIO J 2017;63:14-23.

13. Yarboro LT, Mehaffey JH, Hawkins RB, et al. Pre-implant left ventricular apex position predicts risk of HeartMate II pump thrombosis. J Card Surg 2017;32:837-42.

14. Topilsky Y, Oh JK, Shah DK, et al. Echocardiographic predictors of adverse outcomes after continuous left ventricular assist device implantation. JACC Cardiovasc Imaging 2011;4:211-22.

15. Drakos SG, Janicki L, Horne BD, et al. Risk factors predictive of right ventricular failure after left ventricular assist device implantation. Am J Cardiol 2010;105:1030-5.

16. Topilsky Y, Pereira NL, Shah DK, et al. Left ventricular assist device therapy in patients with restrictive and hypertrophic cardiomyopathy. Circ Heart Fail 2011;4:266-75.

17. Grupper A, Park SJ, Pereira NL, et al. Role of ventricular assist therapy for patients with heart failure and restrictive physiology: Improving outcomes for a lethal disease. J Heart Lung Transplant 2015;34:1042-9.

18. Patel SR, Saeed O, Naftel D, et al. Outcomes of Restrictive and Hypertrophic Cardiomyopathies After LVAD: An INTERMACS Analysis. J Card Fail 2017;23:859-67. 